\title{
Structure determination of soft crystal polymorphs by using prediction technique with powder X-ray diffraction data
}

\author{
S. Obata ${ }^{1}$ and H. Goto ${ }^{1,2}$ \\ ${ }^{1}$ CONFLEX Corporation, Shinagawa Center Bldg. 6F, 3-23-17 Takanawa, Minato-ku, Tokyo, 108-0074, Japan., '2Information and \\ Media Center, Toyohashi University of Technology, 1-1 Hibarigaoka, Tempaku-cho, Toyohashi, Aichi 441-8580, Japan.

$$
\text { obata@conflex.co.jp }
$$

Soft crystals transform into another polymorphic forms by macroscopic gentle stimuli at room temperature and show remarkable changes in luminescence and optical properties [1]. In order to control and analysis the phenomena of soft crystals, it is important to determine the polymorphs and clarify mechanism of the polymorphic transitions. We have developed computational techniques for crystal structure prediction (CSP) and provided successful results in a past blind test of CSP [2]. Recently, we reported a new method related to the CSP technique with measured powder X-ray diffraction (PXRD) data [3]. The method can find the observed crystal structure among a number of computationally suggested structures by using the measured PXRD data and crystal energy, while it is often difficult to find that by only an energy evaluation even with advanced calculations. Therefore, the method can provide candidate structures to experimental crystal structure analyses of unknown crystal structures from the PXRD data.

In this presentation, this new method is applied to flexible organic molecules including the soft crystal materials where the structure analysis from the PXRD data is often difficult, and it is demonstrated that the appropriate crystal structures can be determined by using the CSP technique with PXRD data. We also show that this method is useful for polymorphism analysis.

[1] Kato, M., Ito, H., Hasegawa, M. \& Ishii, K. (2019). Chem. Eur. J. 25, 5105.

[2] Reilly, A. M. et al. (2016). Acta Cryst. B72, 439.

[3] Ishii, H., Obata, S., Niitsu, N., Watanabe, S., Goto, H., Hirose, K., Kobayashi, N., Okamoto, T. \& Takeya, J. (2020). Sci. Rep. 10, 2524.

Keywords: Crystal structure prediction; Powder X-ray crystal structure analysis; Polymorphism

This work was supported by JSPS KAKENHI Grant Number JP17H06373. 